\title{
Variabilité phénotypique de Pseudomonas syringae pv syringae provenant de laurier palme et d'hôtes variés
}

\author{
L Gardan 1, S Cottin 1, C Bollet 2, G Hunault 3, N Boutefnouchet 2 \\ ' INRA, station de pathologie végétale et phytobactériologie, 49070 Beaucouzé; \\ 2 Hôpital Salvator, 249 bd Ste-Marguerite, 13009 Marseille; \\ 3 Faculté des sciences, bd Lavoisier, 49045 Angers, France
}

(Reçu le 22 novembre 1989; accepté le 28 février 1990)

\begin{abstract}
Résumé - La criblure bactérienne du laurier palme (Prunus laurocerasus $L$ ) a été observée pour la 1 re fois en France en 1976; elle est due à Pseudomonas syringae pv syringae. La variabilité phénotypique de 50 souches de $P s$ pv syringae isolées de laurier palme et de 58 souches de $P s$ pv syringae isolées d'hôtes variés a été étudiée. Les résultats ont fait l'objet d'une taxonomie numérique. Les souches de laurier palme, étudiées seules, montrent une certaine variabilité puisqu'elles se répartissent en 6 groupes que l'on peut différencier par 15 caractères biochimiques. Comparées aux autres souches de $P s$ pv syringae, elles se répartissent en 7 groupes caractérisés par 26 caractères biochimiques. Trois souches mal identifiées ne sont pas des $P$ s pv syringae (U 21-1,1787, 1701). La souche type de $P s$ pv syringae a une distance relativement élevée par rapport à la majorité des autres souches.
\end{abstract}

laurier palme / Pseudomonas syringae pv syringae / variabilité / taxonomie numérique / identification / biotypage

Summary - Phenotypic heterogeneity of Pseudomonas syringae pv syringae isolated from cherry laurel and various hosts. Bacterial leaf spot of cherry laurel (Prunus laurocerasus $L$ ) due to $\mathrm{P}$ s pv syringae was observed first in 1976 in France. The phenotypic variability was investigated in 50 representative strains of $\mathrm{P} s$ pv syringae isolated from cherry laurel and 58 strains of $\mathrm{P} s$ pv syringae isolated from 1950 to 1986 from various host-plants classified in 31 genera (tables I and II). The results of 167 biochemical characters were interpreted in numerical taxonomy by the Jaccard and Sneath coefficient. Strains were clustered using the UPGMA method. Strains of P s pv syringae isolated from cherry laurel, analyzed separately, were variable since they were classed in 6 groups (plus 10 isolated phenotypes) that were differentiated by 15 chemical characters (table III and fig 1). Compared with the 58 strains of $\mathrm{P}$ s pv syringae, the strains were distributed among 7 groups (plus 10 isolated phenotypes) which are differentiated by 26 biochemical characters (table $V$ and fig 2). Strains of cherry laurel were clustered in 2 groups with other strains of various host-plants (group 2: 5 strains from cherry laurel plus 1 strain from Phaseolus sp and group 6: 44 strains from cherry laurel and 25 strains from various host-plants). The type strain of $\mathrm{P} s$ pv syringae was very far from the other strains and not a good type strain. A new strain would therefore have to be chosen. Strains clustered in group 6 could be considered as belonging to species $\mathrm{P}$ s pv syringae. However, this would have to be confirmed by DNADNA hybridization. The validity of species $\mathrm{P} s$ pv syringae and the notion of pathovar are discussed.

cherry laurel / Pseudomonas syringae pv syringae / variability / numerical taxonomy / identification / biotyping

\section{INTRODUCTION}

La criblure bactérienne du laurier palme (Prunus laurocerasus $L$ ) a d'abord été décrite au Canada par de Boer (1980). En France, cette maladie a été observée pour la première fois en 1976 dans la région d'Angers, sur des jeunes plants (cv Caucasica) cultivés en plein champ (Gardan et al, 1989).
Les symptômes se manifestent essentiellement sur les feuilles, par des taches vitreuses puis brunes, cernées d'un halo chlorotique jaune, qui évoluent en criblure et sur les rameaux herbacés par des nécroses et des chancres. Les dégâts sont importants sur les jeunes plantes en cours de multiplication. Les criblures et la chute des feuilles peuvent rendre invendables jusqu'à $40 \%$ des plants. Parmi les 
cultivars les plus multipliés en France, le cultivar Caucasica est plus touché que les cultivars Otto Luyken et Rotundifolia.

De 1976 à 1988, 231 souches ont été isolées de lésions et de surface de feuille. Nous présentons d'abord dans cet article, la variabilité des caractères biochimiques des 50 souches isolées de laurier palme, identifiées comme $P s$ pv syringae, puis en comparaison avec les 58 autres souches de $P s$ pv syringae isolées d'hôtes variés.

\section{MATÉRIEL ET MÉTHODES}

\section{Souches}

Cinquante souches de $P$ s pv syringae isolées de laurier palme, représentatives de la collection des 231 souches, ont été retenues d'après leurs origines géographique, variétale et temporelle. La souche type (CFBP $n^{\circ} 1392$ ) sert de témoin.

Cinquante-huit autres souches de $P s$ pv syringae ayant été isolées de 1950 à 1986 en France et à l'étranger, d'hôtes variés classés dans 31 genres botaniques, ont été incluses dans l'étude (tableau I).

\section{Tests biochimiques}

Le caractère hypersensibilité sur tabac et 19 caractères biochimiques ont été étudiés : production de pigment fluorescent aux UV, de lévane, d'une cytochrome c oxydase et d'une arginine dihydrolase, réduction des nitrates, dégradation de l'esculine, de la gélatine, de la pectine, du polypectate à pH 5 et 8,5 , lipolyse du tween 80 , production d'acide à partir de saccharose, sorbitol, érythritol, mannitol, alcalinisation du milieu en présence d'acides organiques : DLlactate, $L(+)$ tartrate et $\mathrm{D}(-)$ tartrate (Gardan et Luisetti, 1982).

L'assimilation de 49 sucres, 49 acides organiques et 49 acides aminés a été étudiée en utilisant les galeries $50 \mathrm{CH}$, LRA 50 AO, LRA 50 AA (Api System, Gardan et al, 1984). La croissance à $24^{\circ} \mathrm{C}$ est observée jusqu'à $6 \mathrm{j}$.

\section{Taxonomie numérique}

Cent soixante-sept caractères ont été étudiés et le codage logique $1 / 0$ a été utilisé. La matrice des distances a été calculée en utilisant le coefficient de Jaccard et Sneath. L'analyse des grappes a été faite en utilisant la méthode des diamètres moyens des grappes (UPGMA = Unweighted pair group method with averages) (Sokal et Sneath, 1963; Sneath et Sokal, 1973).
Un programme d'identification numérique calcule les paramètres d'identification suivants : la fréquence théorique d'apparition du phénotype dans l'espèce (produit des pourcentages de positivité pour les différentes espèces) qui reflète l'atypie du phénotype, et le pourcentage d'identification (rapport de la fréquence théorique d'apparition du phénotype dans l'espèce à la somme des fréquences d'apparition) (Lapage et al, 1973; Bollet et al, 1988). Ceci peut permettre de rattacher un phénotype isolé à un groupe.

La mesure de la quantité d'informations apportée par chaque test est appréciée par le calcul du coefficient de capacité de diagnostic (CCD) (Descamp et Véron, 1981).

Tous les calculs ont été réalisés sur un ordinateur IBM 3090 avec des programmes écrits en Cobol et en Assembleur.

\section{RÉSULTATS}

Nous avons d'abord étudié la variabilité des 50 souches provenant de laurier palme entre elles en comparaison avec la souche type de $P s$ pv syringae puis, dans un $2^{\circ}$ temps, nous les avons comparées aux 58 souches de $P s$ pv syringae isolées d'hôtes variés.

\section{Variabilité des souches isolées de laurier palme}

Le dendrogramme des distances des 51 souches (fig 1) donne une bonne idée de la variabilité des souches; sa coupure à la distance 0,0819 nous donne 6 groupes et 10 phénotypes isolés. L'identification numérique n'a pas permis de rattacher ces phénotypes isolés à un de ces 6 groupes, ce qui paraît logique lorsqu'on examine leur place dans le dendrogramme des distances (fig 1) et qui est confirmé par l'examen de leurs caractères biochimiques comparés à ceux des groupes (tableau II).

Les effectifs des groupes varient de 2 à 15 . Les groupes 6 et 2 sont les plus importants puisqu'ils contiennent respectivement 14 et 15 souches. Les individus sont rassemblés dans chaque groupe du fait de leurs propriétés phénotypiques et aucune relation ne peut être faite entre les individus des groupes et les caractéristiques propres des souches : origine, année d'isolement, nature de l'isolement et cultivar (tableau 1).

La souche type de $P s$ pv syringae (CFBP $n^{\circ}$ 1392) et la souche $U$ 21-1 sont éloignées des autres souches puisqu'elles s'agrègent à l'en- 
Tableau I. Caractéristiques des 58 souches de $P s$ pv syringae isolées de différentes plantes hôtes. * $C F B P$ : Collection française de bactéries phytopathogènes, INRA, Angers, France. NCPPB : National Collection of Plant Pathogenic Bacteria, Harpenden, Royaume-Uni. ** Les numéros de souches précédés d'une lettre proviennent de la collection de laboratoire L Gardan.

\begin{tabular}{|c|c|c|c|c|c|}
\hline Hôte d'isolement & $\begin{array}{l}N^{\circ} \text { de le } \\
C F B P\end{array}$ & $\begin{array}{l}\text { souche * } \\
\text { NCPPB }\end{array}$ & Lieu & Année & $\begin{array}{l}\text { Souche } \\
\text { isolée par }\end{array}$ \\
\hline Allium cepa & 1787 & 2737 & Japon & & Goto M \\
\hline Callistemon viminelis & 1776 & 2734 & Nouvelle-Zélande & 1972 & Wilkie JP \\
\hline Citrus clementina & 1607 & 2781 & France & 1975 & Luisetti J \\
\hline Citrus limon & 1703 & 2307 & Italie & 1968 & Mazzucchi U \\
\hline Citrus sinensis & 1779 & 2786 & Grèce & 1962 & Panagopoulos CG \\
\hline Citrus sp & 1772 & 2814 & États-Unis & 1975 & Lai M, Hildebrand DC \\
\hline Cotoneaster sp & 1773 & 2858 & France & 1975 & Sellwood JE \\
\hline Crataegus sp & 1689 & 1296 & Grande-Bretagne & 1961 & Jones GE \\
\hline Cucurbita maxima & 1788 & 2742 & Nouvelle-Zélande & 1973 & Wilkie JP \\
\hline Cucurbita sp & 1780 & 2804 & États-Unis & 1967 & Schroth MN \\
\hline Delphinium sp & $\mathrm{N} 12.2^{* \star}$ & & France & 1980 & Gardan L \\
\hline Euphorbia pulcherrima & 1777 & 2739 & Nouvelle-Zélande & 1972 & Dye DW \\
\hline Evonymus japonicus & 1542 & 2784 & France & 1974 & Gardan L \\
\hline Forsythia sp & 2576 & & France & 1979 & Gardan L \\
\hline Forsythia sp & M10.5 & & France & 1979 & Gardan L \\
\hline Hibiscus esculentus & 1760 & B1996 & Kenya & 1965 & Hayward AC \\
\hline Hibiscus $s p$ & 1758 & 798 & Australie & 1960 & Magee CJ, Hayward AC \\
\hline Juglans regia & 700 & 2777 & France & 1965 & Luisetti J \\
\hline Lycopersicum esculentum & 1789 & 2749 & Australie & 1970 & Moffett M \\
\hline Magnolia sp & 1678 & 384 & Grande-Bretagne & 1956 & Lelliott RA \\
\hline Magnolia sp & P65.4 & & France & 1981 & Gardan L \\
\hline Malus domestica & 2559 & & France & 1980 & Gardan L \\
\hline Malus sylvestris & 602 & 2775 & France & 1964 & Ride M \\
\hline Olea europea & S43.3 & & France & 1983 & Gardan L \\
\hline Oryza sativa & 1655 & 1417 & Hongrie & 1956 & Klement Z \\
\hline Persea americana & 1701 & 1914 & Nouvelle-Zélande & 1955 & Dye DW \\
\hline Phaseolus vulgaris & 1514 & & États-Unis & 1966 & Natti JJ, Rudolph K \\
\hline Phaseolus vulgaris & 1690 & 1901 & États-Unis & 1920 & Nelson R \\
\hline Pisum sativum & 1765 & 2689 & Nouvelle-Zélande & 1970 & Taylor JD \\
\hline Populus canadensis var eugenei & 1408 & 981 & Grande-Bretagne & 1961 & Dowson WJ \\
\hline Populus sp & 600 & 2776 & France & 1964 & Ride $\mathrm{M}$ \\
\hline Prunus amygdalus & 1577 & 2767 & France & 1974 & Prunier JP \\
\hline Prunus armeniaca & 1578 & 2768 & France & 1974 & Prunier JP \\
\hline Prunus armeniaca & 1588 & & Turquie & 1973 & Rudolph K \\
\hline Prunus avium & 1680 & 1087 & Hongrie & 1958 & Klement $\mathrm{Z}$ \\
\hline Prunus avium & 2006 & & Nouvelle-Zélande & 1974 & Young JM \\
\hline Prunus cerasus & 1543 & & France & 1974 & Gardan L \\
\hline Prunuscerasus & 2334 & & France & 1981 & Prunier JP \\
\hline Prunus domestica & 692 & & France & 1965 & Luisetti J \\
\hline Prunus persica & 1582 & 2779 & France & 1969 & Gardan L \\
\hline Prunus persica & 1584 & & France & 1974 & Prunier JP \\
\hline Prunus triloba & R10.1 & & France & 1982 & Gardan L \\
\hline Prunus triloba & S9.6 & & France & 1983 & Gardan L \\
\hline Pyrus communis & 11 & & France & 1957 & Ride $M$ \\
\hline Pyrus communis & 311 & & France & 1962 & Ride M \\
\hline Pyrus communis & 1391 & 1105 & Grande-Bretagne & 1959 & Billing E \\
\hline Rosa sp & 1608 & 2783 & France & 1975 & Luisetti J \\
\hline Rubus idaeus & 1586 & 2782 & France & 1974 & Luisetti J \\
\hline Salix matsudana var tortuosa & 1692 & 2052 & Grande-Bretagne & 1967 & Lelliott RA \\
\hline Salix sp & 1771 & 2745 & Nouvelle-Zélande & 1968 & Watson DRW \\
\hline Sorghum vulgare var sudanense & 1684 & 2109 & Hongrie & 1967 & Hevesi M \\
\hline Syringa vulgaris & 775 & 2773 & France & 1965 & Luisetti J \\
\hline Syringa vulgaris & 1392 & 281 & Grande-Bretagne & 1950 & Sabet KA \\
\hline Thuya sp & 2575 & & France & 1979 & Gardan L \\
\hline Triticum aestivum & 1767 & 2844 & États-Unis & 1972 & Otta JD \\
\hline Vicia villosa & 1763 & 2678 & États-Unis & 1975 & Ercolani GL \\
\hline Zea mays & 1685 & 2264 & Yougoslavie & 1965 & Arsenijevic M \\
\hline Zea mays & 1894 & & France & 1978 & Samson R \\
\hline
\end{tabular}




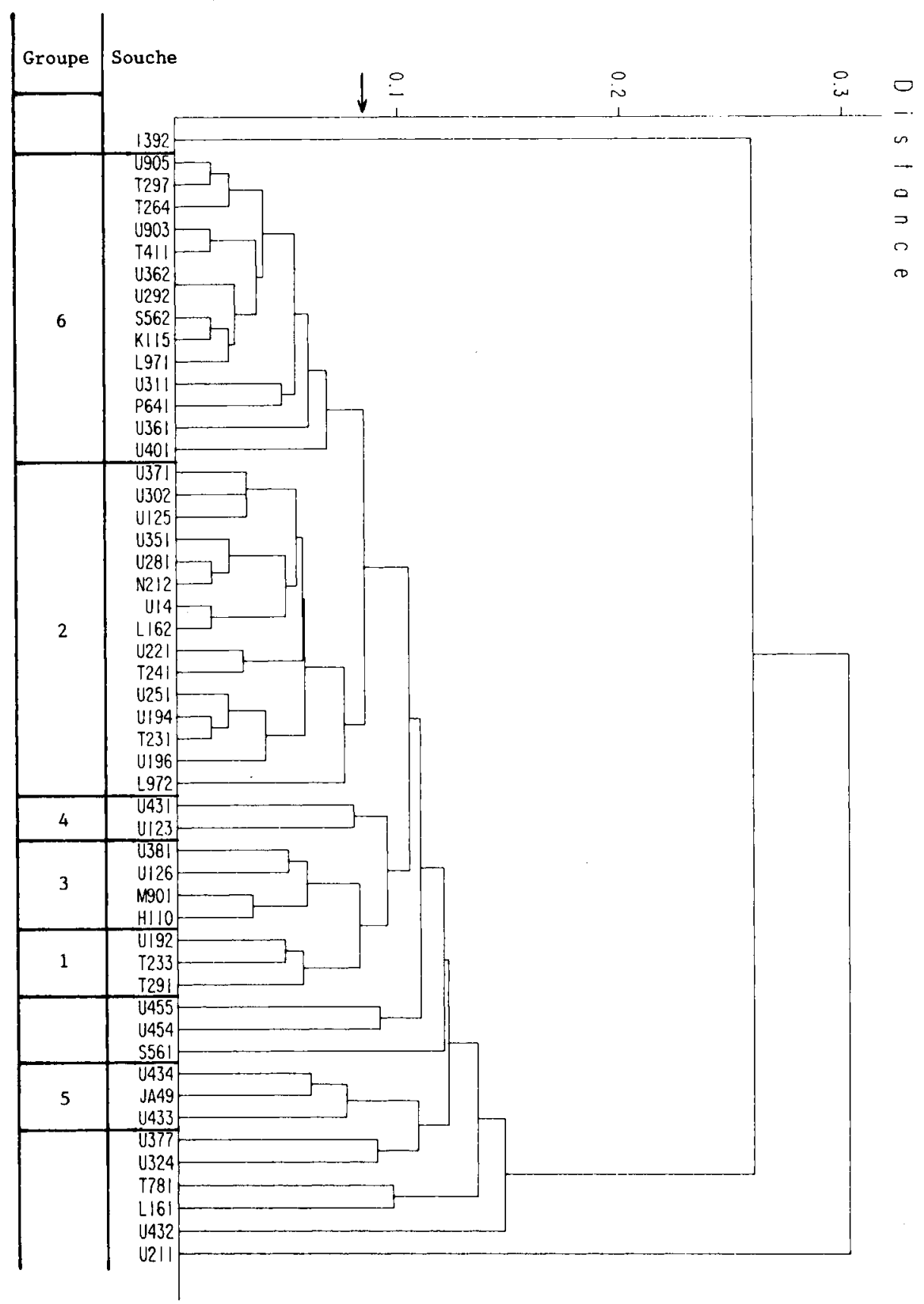

Fig 1. Dendrogramme des distances des 50 souches de $P s$ pv syringae isolées de laurier palme et de la souche type de $P s$ pv $s y-$ ringae (CFBP $\left.n^{\circ} 1392\right)$.

semble des souches à haut niveau, respectivement aux distances 0,259 et 0,302 .

En retenant les CCD les plus élevés de 0,107 à 0,964 , on sélectionne les 15 caractères les plus discriminants qui permettent de différencier les 6 groupes et les phénotypes isolés entre eux (tableau III). Sur la base des caractères malonate et glucosamine on définit l'ensemble des groupes $1+4+5$, le groupe 2 et le groupe 6 .
Le groupe 1 se distingue du groupe 4 (sarcosine, propionate), du groupe 5 (D-xylose) et du groupe 3 (sarcosine).

Le groupe 6 ne s'individualise du groupe 3 que par des caractères notés d (DL-5-aminovalérate, L-cystéine, sarcosine, itaconitate).

Presque toutes les souches sont DL-lactate et 2-cétoglutarate positives et $L(+)$ tartrate négatives. 


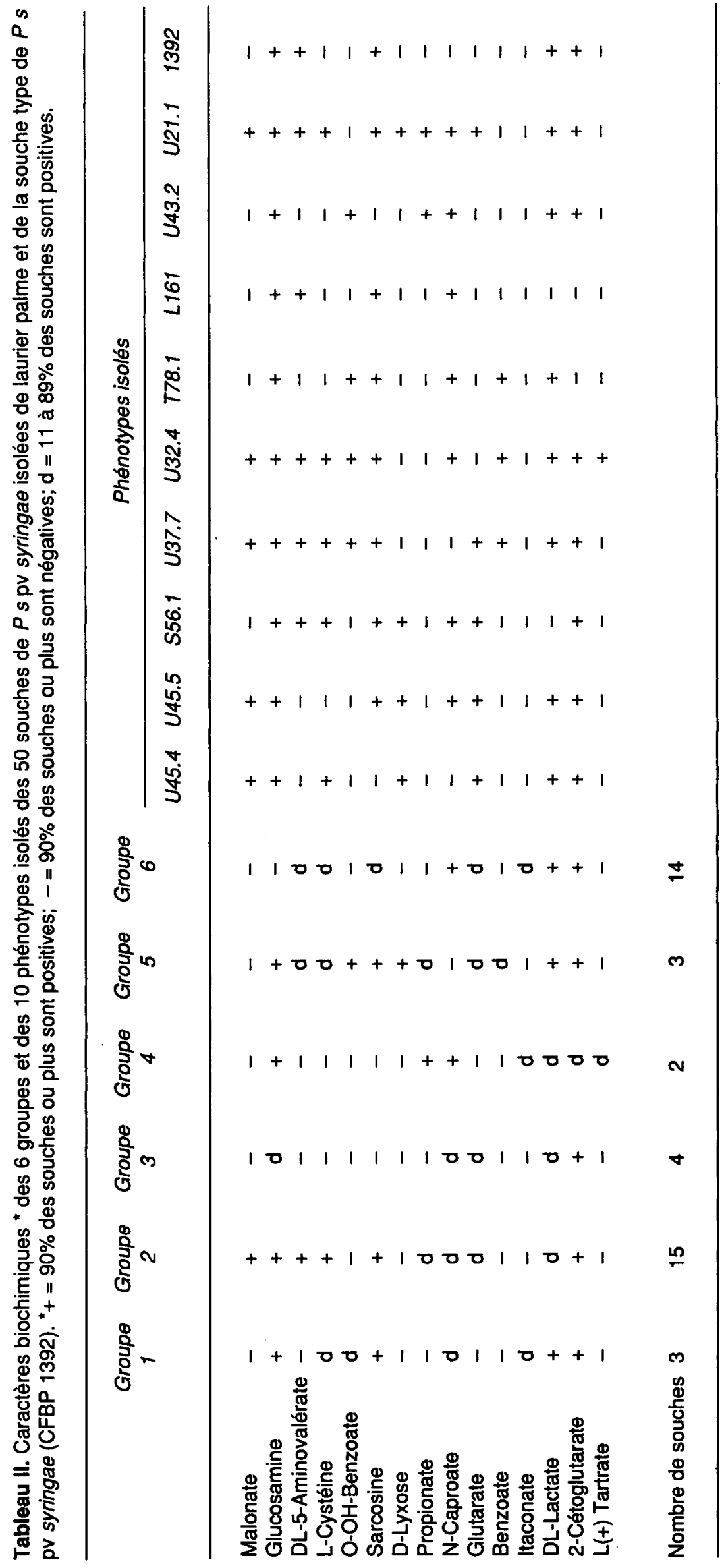


Tableau III. Biotypes selon Hildebrand et Schroth (1972) des différentes souches de $P$ s pv syringae isolées de laurier palme et d'hôtes variés.

\section{Sorbitol Erythritol DL Lactate $L(+)$ Tartrate $D(-)$ Tartrate Nombre de souches \%}

Biotypes des 50 souches de $\mathrm{P}$ s pv syringae, isolées de laurier palme

\begin{tabular}{|c|c|c|c|c|c|c|c|}
\hline 1 & + & + & - & - & + & 1 & 2 \\
\hline 2 & + & + & + & - & - & 1 & 2 \\
\hline 3 & + & + & + & - & + & 45 & 90 \\
\hline 4 & + & + & + & + & + & 2 & 4 \\
\hline 5 & - & - & + & - & + & 1 & 2 \\
\hline
\end{tabular}

Biotypes des 58 souches de P s pv syringae, isolées d'hôtes différents

\begin{tabular}{rrrrrrrr}
1 & + & + & - & - & + & 3 & 5 \\
2 & + & + & + & - & - & 11 & 19 \\
3 & + & + & + & - & + & 37 & 64 \\
6 & + & - & + & - & + & 6 & 2 \\
7 & + & - & - & - & + & 1 & 10 \\
\hline
\end{tabular}

\section{Variabilité comparée des souches de $\mathrm{P}$ s pv syringae isolées de laurier palme et des autres hôtes}

Le typage sur la base de 5 caractères classiques de Hildebrand et Schroth (1972) (sorbitol, érythritol, DL-lactate, $\mathrm{L}(+)$ tartrate, $\mathrm{D}(-)$ tartrate) donne 7 biotypes au total (tableau III). La répartition de l'ensemble des souches dans les différents biotypes montre que le biotype 3 est majoritaire puisqu'il rassemble $90 \%$ des souches isolées de laurier palme et $64 \%$ des souches provenant de différents hôtes. Le biotype 2 est encore conséquent pour les souches isolées d'hôtes variés puisqu'il rassemble 19\% des souches; par contre les autres biotypes sont minoritaires puisqu'ils représentent moins de $10 \%$ des souches des 2 provenances.

La souche type de $P s$ pv syringae (CFBP $n^{\circ}$ 1392) est classée dans le biotype 2 et non dans le biotype majoritaire 3 .

Le dendrogramme (fig 2) montre la répartition des 108 souches suivant les distances qui les séparent. La coupure du dendrogramme à la distance 0,130 fait apparaître 7 groupes et 10 phénotypes isolés (tableau IV).

Le groupe 1 , où figurent 2 répétitions de la souche type de $P s$ pv syringae, est le plus éloigné de l'ensemble des autres groupes.
Les souches provenant de laurier palme sont réparties dans le groupe 6 (44) et le groupe 2 (5). Les souches provenant d'hôtes variés sont réparties de façon plus large (22 dans le groupe 6 et 1 seule dans le groupe 2); elles représentent la totalité des groupes $3,4,5$ et 7 qui ne comprennent que des souches isolées d'hôtes variés. L'identification numérique pourrait permettre de rattacher la souche N 12-2 (isolée de Delphinium $s p$ ) au groupe 7 puisqu'elle en est très proche; ce n'est pas le cas des 9 autres phénotypes isolés.

En retenant les CCD de 0,334 à 0,112 , on sélectionne 25 caractères discriminants qui permettent de bien différencier les 7 groupes entre eux et les 10 phénotypes isolés (tableau IV).

Les caractères glycine, malonate et érythritol permettent de différencier les groupes 1 et 2 des autres groupes.

Le groupe 1 se différencie du groupe 2 par les caractères mésotartrate, L-arginine, succinate, Lhistidine et fumarate.

Les groupes 4 et 6 ont respectivement les caractères glycine et malonate $d$, donc positifs, ou négatifs. Le groupe 4 se différencie du groupe 5 (sarcosine, gélatine, propionate, salicine et Lleucine), du groupe 6 (D-tartrate, L-valine) et du groupe 7 (D-raffinose et L-valine).

Le groupe 6 se distingue du groupe 1 (mésotartrate, L-arginine, succinate, glutarate, L- 


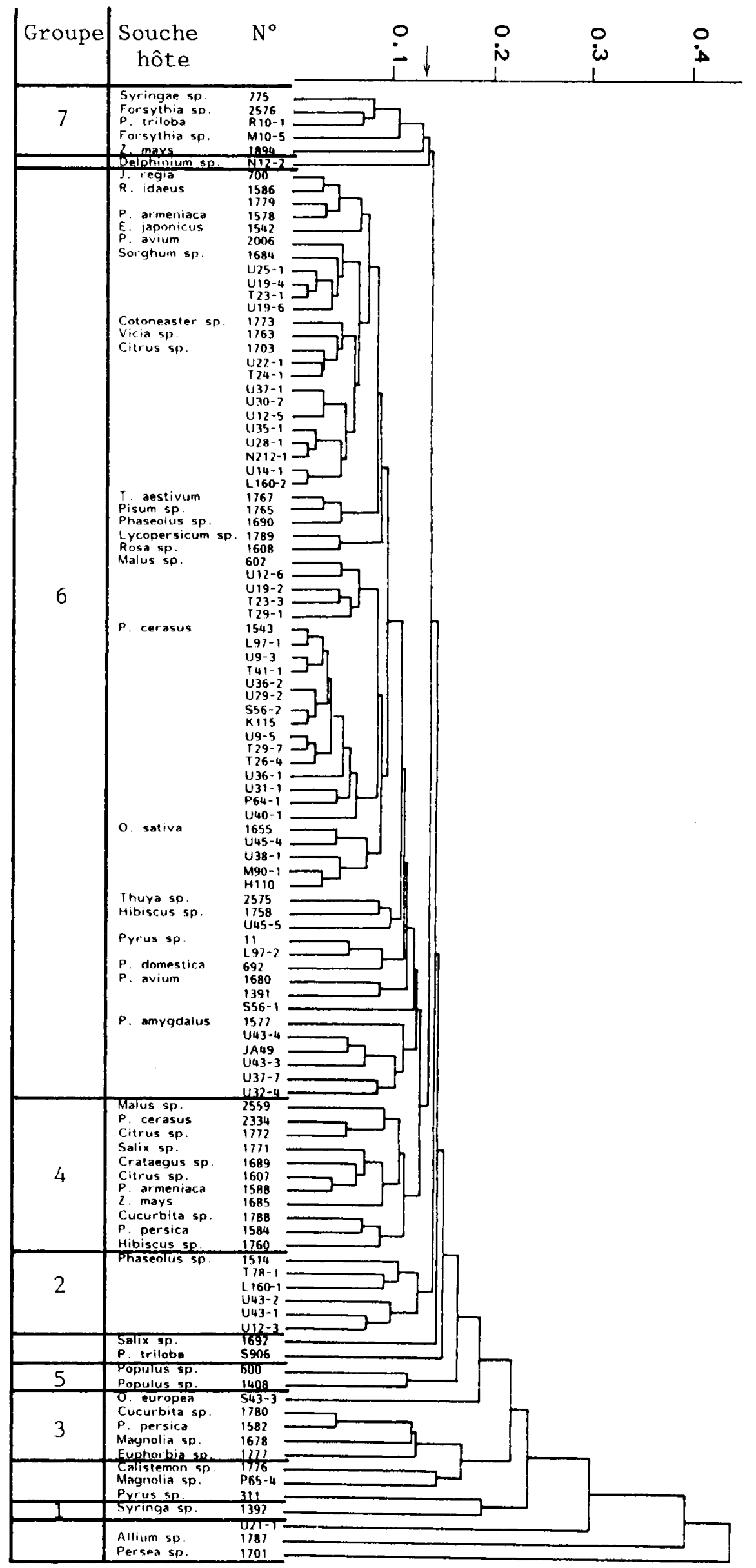

Fig 2. Dendrogramme des distances des 50 souches de $P s$ pv syringae isolées de laurier palme et des 58 souches de $P s$ pv syringae isolées d'hôtes variés. 


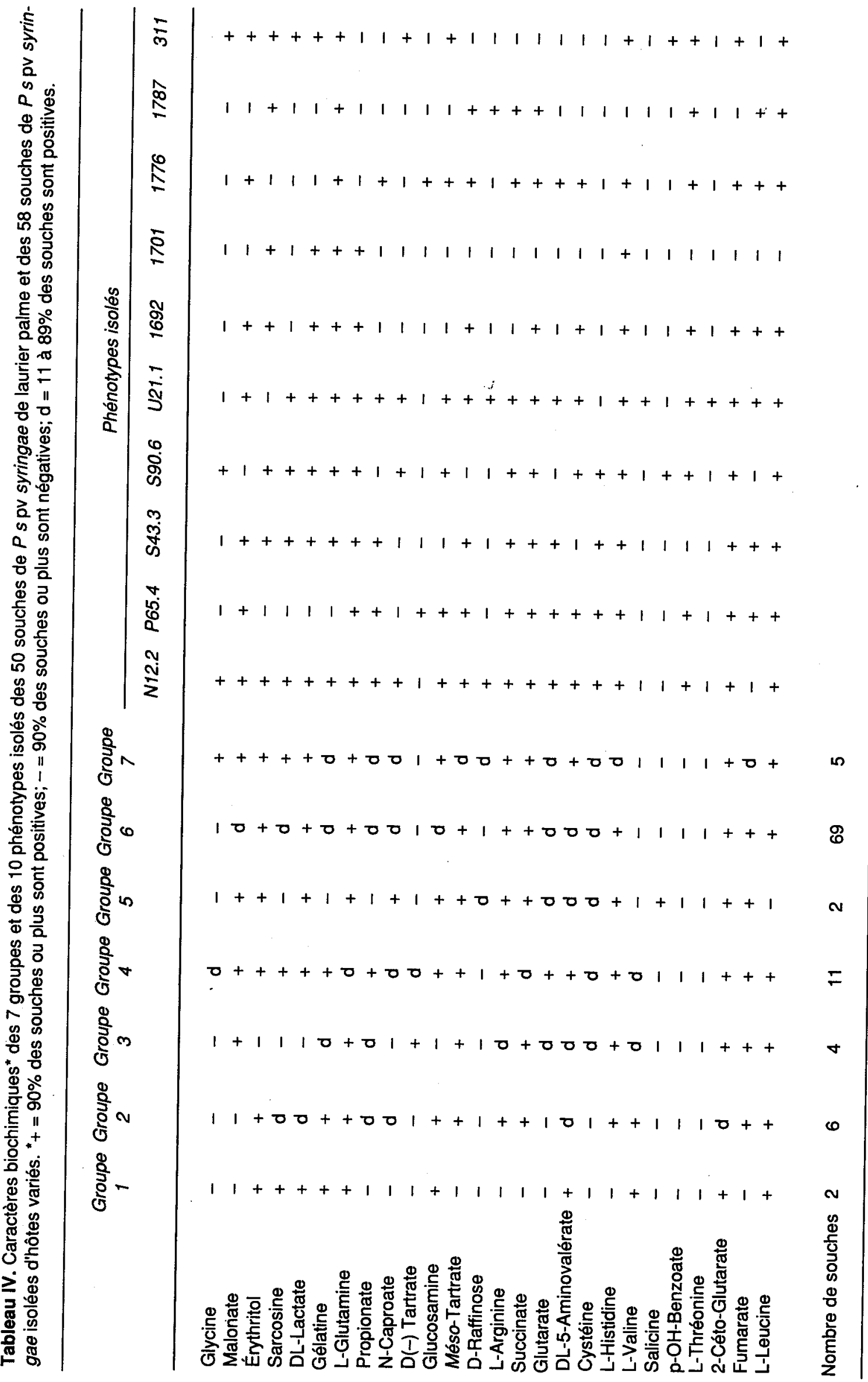


cystéine, L-histidine, L-valine et fumarate), du groupe 2 (glutarate, L-cystéine et L-valine), du groupe 5 (salicine, L-leucine).

Les phénotypes isolés ont des caractères différents les uns des autres et se distinguent facilement des 6 groupes par 1 ou plusieurs caractères (tableau IV).

Les biotypes de Hildebrand et Schroth (1972) et les groupes phénotypiques que nous avons délimités ne coïncident pas; l'ensemble des 69 souches du groupe 6 appartiennent au biotype 3 mais les autres biotypes sont répartis au hasard parmi les groupes phénotypiques.

\section{DISCUSSION}

Les souches isolées de lésions ou à la surface des feuilles de laurier palme et identifiées comme $P s \mathrm{pv}$ syringae ont des caractères biochimiques variés. Ces 50 souches ont des caractères biochimiques que ne semblent pas en relation avec la provenance géographique, la date d'isolement, le cultivar, le type de lésions ni le pouvoir pathogène sur l'hôte homologue.

Comparées à d'autres souches de $P s \mathrm{pv} s y$ ringae isolées d'hôtes variés, elles sont classées pour la majorité dans le groupe 6 (44 souches) mais aussi dans le groupe 2 (5 souches) (fig 2). Ces dernières, quoique isolées de lésions, sont pathogènes ou non sur l'hôte homologue.

Les groupes 2, 4, 6 et 7 pourraient correspondre en fait à l'espèce $P s \mathrm{pv}$ syringae; pour le confirmer il serait nécessaire d'examiner leurs positions relatives par rapport aux autres pathovars de $P$ syringae.

Le choix des caractères biochimiques définissant chaque groupe est facilité par le calcul, pour chaque test, du coefficient de capacité de diagnostic (CCD). La présence d'un certain nombre de caractères notés $d$ nous a obligés à retenir un plus grand nombre de caractères biochimiques.

Sur la base des caractères de Hildebrand et Schroth (1972), la variabilité des caractères biochimiques d'un plus ou moins grand nombre de souches de $P s$ pv syringae provenant d'abricotier (Gardan et al, 1973), de pêcher (Dowler et Weaver, 1973) et de vigne (Luisetti et Gaignard, 1987) a été mise en évidence.

Dans notre étude il n'y a pas de corrélation entre le biotypage réalisé selon Hildebrand et Schroth (1972) et les groupes phénotypiques obtenus en taxonomie numérique. Le système de biotypage que nous proposons, utilise 15 tests au lieu de 5, ce qui pourrait lui donner une assise génomique plus grande.

La souche référence de $P s$ pv syringae isolée de lilas il y a 40 ans est éloignée de l'ensemble des autres souches étudiées : ce n'est donc pas une bonne souche type et il faudrait choisir une autre souche médiane ou centrotype au sein du groupe 6 (Véron, 1982).

Certaines souches sont abusivement identifiées comme $P \boldsymbol{s}$ pv syringae c'est le cas de 3 phénotypes isolés : $U$ 21-1, 1787 et 1701 mais probablement aussi d'autres phénotypes isolés et des souches des groupes 1, 3 et 5 .

"Le terme de pathovar définit des souches ou des groupes de souches ayant des caractéristiques identiques ou similaires, différenciées à un niveau infraspécifique d'autres souches de la même espèce ou sous-espèce sur la base d'une pathogénicité distincte sur un ou plusieurs hôtes" (Young et al, 1978; Dye et al, 1980). Ce concept a été utilisé pour classifier les Pseudomonas fluorescents phytopathogènes des groupes 1 et 2 de Lelliott et al (1966) isolés d'hôtes variés sans que le pouvroir pathogène croisé sur les autres hôtes ait été vérifié.

II semble que plusieurs de ces pathovars soient, en fait, des espèces taxonomiques : $P$ syringae pv savastanoï est ainsi phénotypiquement distinct des autres pathovars; en effet son pourcentage d'hybridation ADN/ADN avec $P s$ pv syringae est de $43 \%$, réciproquement ce dernier s'hybride à $60 \%$ avec $P$ s pv savastanoï (Gardan et al, 1987).

Une étude taxonomique basée sur les caractères phénotypiques et les hybridations ADN/ ADN de tous les pathovars de $P$ syringae permettra seule de clarifier la situation.

\section{RÉFÉRENCES}

Bollet C, Gullian C, Chaudet H, Fichet B, Aragon A, de Micco $P$ (1988) Identification des principales espèces du genre Serratia par une galerie simplifiée. Ann Inst Pasteur/Microbiol 139, 337-349

de Boer SH (1980) Leaf spot of cherry laurel caused by Pseudomonas syringae. Can J Plant Pathol 2, 235-238

Descamps $P$, Véron $M$ (1981) Une méthode de choix des caractères d'identification basée sur le théorème de de Bayes et la mesure de l'information. Ann Inst Pasteur/Microbiol 132B, 157-170

Dowler WM, Weaver DJ (1973) Characterization of green fluorescent Pseudomonas isolated from apparently healthy dormant peach trees. Journées 
d'études sur les bactéries du groupe Pseudomonas syringae, Angers, France, 91-94

Dye DW, Bradbury JF, Goto A, Hayward AC, Lelliott RA, Schroth MN (1980) International standards for naming pathovars of phytopathogenic bacteria and a list of pathovar names and pathotype strains. Rev Plant Pathol 59, 4, 153-168

Gardan L, Luisetti J (1982) Méthodes d'isolement et d'identification des bactéries phytopathogènes, ENITH Angers, $32 p p$

Gardan L, Prunier JP, Luisetti J, Belzegues JJ (1973) Études sur les bactérioses des arbres fruitiers. VII Responsabilité de divers Pseudomonas dans le dépérissement bactérien de l'Abricotier en France. Rev Zool Agric Pathol Vég 4, 112-120

Gardan L, Morando B, Guinebretière JP (1984) The use of a micromethod of auxanogram and data analysis for identification of Pseudomonas. Proc of the 2nd Working group on Pseudomonas syringae pathovars, Athènes, 37-39

Gardan L, Abu Ghorrah M, Grimont PAD, Grimont F (1987) Identification and pathogenicity of Pseudomonas syringae pv savastanoi. In: Proc of 3rd international Working group on Pseudomonas pathovars, Lisbonne, 6-8

Gardan L, Digat B, Krauze A, Cottin S, Lemaire F (1989) La criblure bactérienne du laurier palme. Pépiniéristes Horticulteurs Maraîchers, 294, 29-35
Hildebrand DC, Schroth MN (1972) Identification of the fluorescent Pseudomonads. In: Proc of the 3rd Intern Conf on Plant Pathogenic Bacteria, Wageningen, 14-21 Avril 1971, 281-287

Lapage SP, Bascomb S, Wilcox WR, Curtis MA (1973) Identification of bacteria by computer: general aspects and perspectives. J Gen Microbiol 77, 273290

Lelliott RA, Billing E, Hayward AC (1966) A determinative scheme for the fluorescent plant pathogenic Pseudomonads. J App/ Bacteriol 29, 470-489

Luisetti J, Gaignard JL (1985) Bacterial populations in buds and probability of frost injury on grape vine. In: 6th Intern Conf on Plant Pathogenic Bacteria (Civerolo EL, ed) 983-993

Sneath PHA, Sokal RR (1973) Numerical taxonomy. The principles and practice of numerical classification. Freeman, San francisco

Sokal RR, Sneath PHA (1963) Principles of numerical taxonomy. Freeman, San Francisco

Véron M (1982) Taxonomie bactérienne (L Le Minor, $M$ Véron, eds). Flammarion, Paris, 80-97

Young JM, Dye DW, Bradbury JF, Panagopoulos CG, Robbs CF (1978) A proposed nomenclature and classification for plant pathogenic bacteria. NZJ Agric Res 21, 153-177 